\title{
An Enablement Detection Algorithm for Open Multiparty Interactions
}

\author{
J.A. Pérez, R. Corchuelo, D. Ruiz, and M. Toro \\ Dept. Lenguajes y Sistemas Informáticos \\ Universidad de Sevilla \\ (jperez,corchu,druiz,mtoro\}@Isi.us.es
}

\begin{abstract}
Coordination amongst an arbitrary number of entities has become an important issue in recent years in fields such as ecommerce, web-based applications and so on. Traditionally, classical client/server primitives have been used to implement synchronisation and communication. But, when more than two entities need to coordinate by means of those primitives, the coordination must be decomposed into a number of client/server biparty interactions, leading the programmer to the need of thinking in terms of the protocols needed to achieve properties like livenes, atomicity and so on. In this paper, we present an algorithm to perform enablement detection to implement open multiparty interactions. This primitive provides a high level of abstraction since the programmer can implement multiparty coordination without the need of thinking in terms of protocols.
\end{abstract}

Keywords: Multiparty interactions, coordination algorithms.

\section{INTRODUCTION}

In recent years, the development of distributed applications has been paid much attention, mainly due to the Internet boom. Traditionally, most services provided by means of the network involved only two entities, a provider entity and a client entity. For example, a purchase trough the web involved just two entities: a seller and a purchaser. Recently, more complicated scenarios have emerged, since frequently several entities collaborate to provide a service. For example, in a purchase through the web, the purchaser may order his or her bank to transfer the sale amount to the seller bank account. And both seller and purchaser may require each other to certificate their identity, involving then certification entities in the scenario.

When more than two entities need to collaborate to achieve a common goal, coordination becomes an important issue. Traditionally, coordination has been achieved by means of client/server primitives (remote procedure call, message pass-

Permission to make digital or hard copies of all or part of this work for personal or classroom use is granted without fee provided that copies are not made or distributed for profil or commercial advantage and that copies bear this notice and the full citation on the first page. To copy otherwise, to republish, to post on servers or to redistribute to lists, requires. prior specific permission and/or a fee.

SAC 2002, Madrid, SPAIN

Copyright 2002 ACM 1-58113-445-2/02/03 ...\$5.00. ing, and so on). Those primitives are biparty because they only involve two entities that need to synchronise before exchanging data, but this concept can be easily extended to an arbitrary number of entities that need to agree and cooperate to achieve a common goal. These interactions are usually said to be multiparty, and they provide a higher level of abstraction because they allow to express complex cooperations as atomic units. A taxonomy of languages offering linguistic support for multiparty interactions can be found in [6]. We think that those interaction models are interesting because they allow to express coordination regardless of the protocols needed to achieve it.

Most interaction models proposed in the literature are aimed at coordinating a set of entities that must be known in advance, i.e., they are static models. Those models are not adequate for open scenarios such as e-commerce where frequently entities need to collaborate without knowing one another. For example, in the purchase through the web, nor the purchaser knows the account of the seller, neither vice versa. Furthermore, the seller do not need to know the client in advance.

In [2], the CAL language is presented. The CAL language is aimed at increasing the level of abstraction of a programme by considering the concurrent behaviour of components as an aspect where multiparty interactions are the sole means for synchronisation and communication. CAL relies on an open interaction model that allows to express coordination amongst entities that do not know one another in advance.

Although several authors have proposed algorithms to implement multiparty interactions as a coordination primitive $[9,1,5,4,6]$, they have focused on finding solutions to achieve exclusion in the scope of static interaction models. To implement an open, dynamic interaction model we must deal not only with the exclusion problem, but also with the enablement detection problem. The enablement detection consists on finding sets of entities that agree to coordinate through a given interaction. In an open context, this is an important problem since in general, it has a high computational cost.

In this paper we present the algorithms we have devised to implement enablement detection in the CAL interaction model. The problem of finding enablements in this model has a computational combinatorial complexity. But, by means of the adequate data structures, the algorithms we propose behave quite efficiently in most practical situations. Furthermore, they can be customized with a selection algo- 


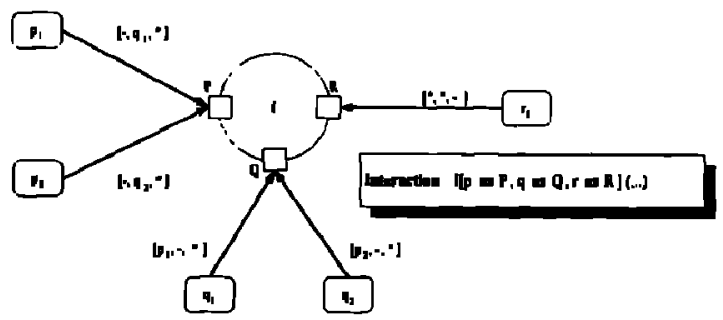

Figure 1: Example of CAL interaction model.

rithm to solve the exclusion problem.

The rest of this paper is organised as follows: section 2 sketches the CAL interaction model; section 3 outlines our strategy to implement this model; section 4 focuses on the algorithms we have developed to perform enablement detection, and section 5 glances at the problem of enablement selection. Next, we show some performance results from our implementation in section 6, and finally, section 7 shows our conclusions.

\section{AN OPEN MULTIPARTY INTERACTION MODEL}

This section presents the CAL [2] interaction model. To the best of our knowledge, this is the only open, dynamic interaction model proposed so far. It improves static models in that it can coordinate entities that do not need to know one another. This is very important because it makes it feasible as a coordination primitive for open systems. As we describe in this section, interactions in CAL work as templates of static interactions.

In CAL, each interaction is given a name, a number of roles and a number of slots associated with it. The name of the interaction is a string which unambiguously identifies an interaction in the system. When an object is ready to coordinate with other objects, it offers to participate in one or more interactions by means of their names.

So, every object can offer participation in one or more interactions simultaneously. In every offer, a participant states which role it plays in the interaction, and may establish constraints on what objects should play the other roles. An interaction may be executed as long as a set of objects satisfying the following constraints is found: (i) there is an object per role willing to participate in that interaction and play that role; (ii) those objects agree in interacting with each other, i.e., the constraints they establish are satisfied. A set of objects which can execute an interaction is what we call an enablement.

Figure 1 shows an example with an interaction called $I$ amongst three objects that must play roles $P, Q$ and $R$. Objects $p_{1}$ and $p_{2}$ make offers to play role $P$, objects $q_{1}$ and $g_{2}$ make offers to play role $Q$, and object $r_{1}$ makes an offer to play role $R$. The objects $p_{1}$ and $p_{2}$ require that role $Q$ must be played by $q_{1}$ and $q_{2}$ respectively, and vice versa. Neither $p_{1}, q_{1}, p_{2}$ nor $q_{2}$ establish constraints on what object should play role $R$. On the other hand, the object $r_{1}$ accepts that roles $P$ and $Q$ can be played by any object.

Since exclusion must be guaranteed, an object cannot commit to more than one interaction at a time. But, since an object can offer participation simultaneously in more than one interaction, it can be in more than one enablement. So, when two or more enablements share objects, they cannot be executed simultaneously. The set of enablements that cannot be executed are refused.

When an enablement of an interaction is executed, the objects in it can communicate by means of the interaction slots. A slot is a shared variable amongst the objects in the enablement which is created when the enablement is executed. These slots make up a local state that simulates the temporary global combined state in IP [3], being the most important difference that an object does not need to have access to the local state of other objects in order to get the information it needs. Obviously, a multiparty interaction delays an object that tries to read a slot that has not been initialized yet by another object.

\section{STRATEGY TO IMPLEMENT THE CAL INTERACTION MODEL}

In this section, we describe the strategy we have used to implement the CAL multiparty interaction model. We follow a "divide-and-conquer" strategy, since we split the execution of an interaction into two steps:

Synchronisation: The execution of an interaction begins when a set of entities get synchronised and commit to the interaction. This synchronisation can also be divided into two steps:

Enablement detection: The participation offers are analysed to find sets of objects that agree in participating in an interaction, i.e, enablements.

Enablement selection: When one or more enablements have been detected, as many as possible of them should be executed simultaneously, ensuring exclusion. Thus, an election under conflicting enablements needs to be held.

Communication: Once an enablement of an interaction having slots has been selected to execute, a set of slots must be instatiated for it. The details concerning communication fall beyond the scope of this paper.

The algorithm we have developed to implement synchronisation is called $\alpha$. This algorithm uses a special resource per interaction, called interaction coordinator. This is an object which receives the offers of participation in a given interaction issued by entities in the system.

Enablement detection may be performed in a local manner in the interaction coordinator. In other words, a coordinator can compute the enablements originated by a set of offers made to it disregarding the offers made to other coordinators of potentially conflicting interactions. When the enablement detection algorithm finds out one enablement, this enablement is dealt with as if it was an entity which must compete to achieve exclusion over every participant in it. This task is performed by an enablement selection algorithm.

\section{ENABLEMENT DETECTION}

In this section we describe $\alpha$-solver, which is the algorithm we have devised to implement enablement detection in the scope of $\alpha$. $\alpha-s o l v e r$ builds a data structure which holds the information about every offer being processed. This data 
structure is updated (i) every time an offer is received by the coordinator and (ii) every time that an offer is given up because the participant that made it is executing an interaction. The latest may happen either because an enablement of the interaction has been selected for execution, or because an enablement of another conflicting interaction has been selected for execution.

\subsection{Data structures}

Consider for example a system like the one in Figure 1 with an interaction called $I$ amongst three objects that must play roles $P, Q$ and $R$. Assume that objects $p_{1}$ and $p_{2}$ make offers to play role $P$, objects $q_{1}$ and $q_{2}$ make offers to play role $Q$ and that object, $r_{1}$ makes an offer to play role $R$.

$\alpha$-salver uses an acyclic directed graph to store the information about the offers being processed. Every node in the graph holds a data structure that we call tuple, such as $\left[p_{1},\left(q_{1}\right),()\right]$. This tuple represents the offer made by $p_{1}$ and it means that it wants to play role $P$ in interaction $I$, requires $q_{1}$ to play role $Q$, and does not care about which object should play role $R$. We say that role $P$ is consolidated in this tuple, whereas role $Q$ requires object $q_{1}$ and role $R$ accepts any object. So, when an offer is received, a tuple with its information is created for it, and the graph is updated with this tuple and probably with other tuples calculated from it. Some of this calculated tuples may stand for an enablement. Furthermore, when the participant that made the offer commits to an interaction, the tuple which holds the information related to such offers is removed from the graph, and the tuples calculated from it are removed or updated to take this change into account.

Figure 2 shows the graph built by our algorithm as the offers made by the objects in our example arrive at the coordinator responsible for interaction $I$. Assume that the offer made by $p_{1}$ arrives first so that $\alpha-s o l v e r$ constructs a graph with only one node $\left[p_{1},\left(q_{1}\right),()\right]$. If the second offer is made by object $p_{2}$, a new node of the form $\left[p_{2},\left(g_{2}\right),()\right]$ is added to the graph, and no connecting node is constructed because the tuples so far processed are not compatible, i.e., objects $p_{1}$ and $p_{2}$ cannot interact together. If the offer made by $q_{1}$ is then received, a node of the form $\left[\left(p_{1}\right), q_{1}, 0\right]$ is added. Since it is compatible with $\left[p_{1},\left(q_{1}\right),()\right]$, a connecting node of the form $\left[p_{1}, q_{1},()\right]$ is added. It indicates that both $p_{1}$ and $q_{1}$ want to participate in interaction $I$ and agree in committing to it together with any object playing role $R$. Notice that no enablement is found until object $r_{1}$ makes its offer. When this happens, two enablements are found simultaneously, but they are conflicting because they share $r_{1}$.

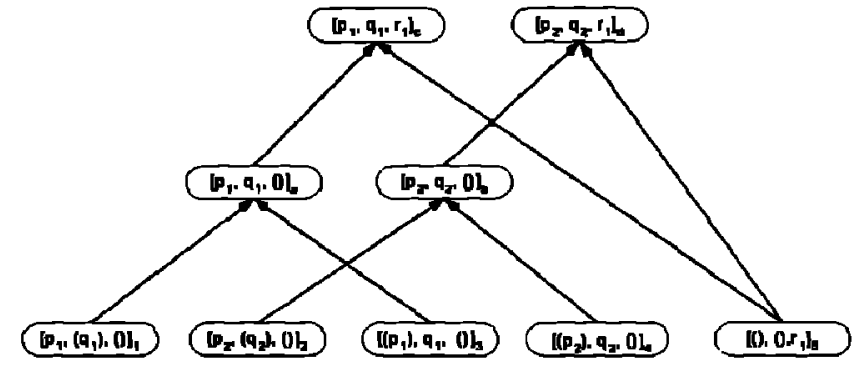

Figure 2: Consolidation graph for the system in Figure 1.
In order to formalise the concept of compatibility amongst tuples, we define a consolidation operator that is defined on both the tuples of the graph and its elements. We refer to this operator as $\odot$ and it is defined on tuples as $\left[e_{1}, e_{2}, \ldots, e_{n}\right] \odot\left[e_{1}^{\prime}, e_{2}^{\prime}, \ldots, e_{n}^{\prime}\right]=\left[e_{1} \odot e_{1}^{\prime}, e_{2} \odot e_{2}^{\prime}, \ldots, e_{n} \odot e_{n}^{\prime}\right]$. It is defined on the elements of a tuple by means of the following axioms:

$$
\begin{aligned}
& \text { 1. } p_{1} \odot\left(p_{2}\right)=\left(p_{2}\right) \odot p_{1}=p_{1}, \text { as long as } p_{1}=p_{2} \\
& \text { 2. }\left(p_{1}\right) \odot\left(p_{2}\right)=\left(p_{2}\right) \odot\left(p_{1}\right)=\left(p_{1}\right) \text {, as long as } p_{1}=p_{2} \\
& \text { 3. } p \odot()=0 \odot p=p \\
& \text { 4. }(p) \odot O=0 \odot(p)=(p) \\
& \text { 5. }() \odot 0=0
\end{aligned}
$$

Note that this operation is defined on two tuples if and only if both tuples represent offers that can lead to an enablement. Furthermore, if the $\odot$ operation is defined between two tuples, the resulting tuple holds the combined information of the consolidated tuples. For example, this is the case of $\left[\left(p_{1}\right), q_{1},()\right]$ and $\left[p_{1},\left(q_{1}\right),()\right]$ in Figure 2 . The consolidation of those tuples is a tuple $\left[p_{1}, q_{1},()\right]$, which means that $p_{1}$ wants to play role $P$ in the interaction, that $q_{1}$ wants to play role $Q$, and that both of them accept that role $R$ can be played by any object. Furthermore, the consolidation operation is not defined on tuples $\left[p_{1},\left(g_{1}\right),()\right]$ and $\left[p_{2},\left(q_{2}\right),()\right]$, because they are incompatible since both $p_{1}$ and $q_{1}$ are willing to play role $P_{1}$ and $p_{1}$ requires that role $Q$ be played by $q_{1}$ and $p_{2}$ requires the same role to be played by $q_{2}$. Since the graph is built with consolidation amongst the tuples which represent the offers, we usually refer to it as the consolidation graph. In this graph, we refer to the top-most tuples (having no outgoing edge) as roots, and we refer to the bottom-most tuples (with no incoming edge) as leaves.

\subsection{Processing offers}

Figure 3 shows a routine called Process Of fer $(T, G)$. This routine is the entry-point to $\alpha$-solver. Parameters $T$ and $G$ represent the offer being processed and the current consolidation graph, respectively. It simply iterates over the set of roots of graph $G$ and calls routine $\operatorname{Search}(T, R)$ (presented in the same figure) on each one. Its parameters $T$ and $\boldsymbol{R}$ represent the current offer and the root where search begins, respectively. This routine first tries to consolidate tuples $\boldsymbol{T}$ and $R$, and if it is possible, the consolidated tuple is returned and inserted in the graph as a parent of both $T$ and $R$. Otherwise, a recursive search is performed in the subgraph whose root is the left child of $R$. If a consolidation left is found there, it recursively tries to find out a new consolidation of left with a tuple in the subgraph whose root is the right child of $\boldsymbol{R}$. If such a consolidation if found, then it is returned because it is the most consolidated tuple that has been found; else, left is returned. If no consolidation is found while examining the left subgraph of $R$, then the right subgraph is also explored. If no consolidation is found, then null is returned.

\subsection{Offer cancellation processing}

As we stated before, when an object commits to an interaction, the offers it made give up being valid. So, the information related to them must be removed from the consolidation graph. 
For example, assume that participant $p_{1}$ in Figure 1 commits to another interaction. Then, the tuple $\left[p_{1},\left(q_{1}\right),()\right]$ must be removed from the consolidation graph in Figure 2. If this tuple was just removed from the graph, it would result in an inconsistent state since the tuple $\left[p_{1}, q_{1},()\right]$ would bave one only descendant. Every tuple in the consolidation graph has either two or zero descendants, since the $\odot$ operator is a binary operator.

Figure 4 shows a routine called Process $\operatorname{Cancel}(P, G)$. This routine is the entry-point to the $\alpha$-golver offer cancellation algorithm. Parameters $P$ and $G$ are the participant whose offers are canceled and the current consolidation graph, respectively. The ProcessCancel routine iterates over the set of leaves of graph $G$ having object $P$ in consolidated state (in other words, the offers made by $P$ ), and calls routine $\operatorname{Delete}(T, R)$ on each one. Its parameter $T$ represents the offer being deleted. The deletion algorithm consists of (i) replacing every parent of the tuple being deleted by the other descendant of the parent (we assume that $\operatorname{Brother}(T, p)$ returns the other descendant that $p$ of tuple $T$ ) and (ii) calling a recursive routine Rebuild(T) (Figure 5) which recursively re-consolidates the ancestors of tuple $T$.

The full cancellation process for the offers of $p_{1}$ is sketched in Figure 6. The only leaf having $p_{1}$ in consolidated state is $\left[p_{1},\left(q_{1}\right),()\right]$, so this tuple is passed to routine Delete as argument $T$. This algorithm first (1) replaces tuple $\left[p_{1}, g_{1},()\right]$ by its other descendant, the tuple $\left[\left(p_{1}\right), g_{1},()\right]$. Since this would left the graph into an inconsistent state, the Rebuild routine reconsolidates (2) the tuple $\left[(),(), r_{1}\right]$ with $\left[\left(p_{1}\right), q_{1},()\right]$, giving a tuple $\left[\left(p_{1}\right), q_{1}, r_{1}\right]$ which replaces $\left[p_{1}, q_{1}, r_{1}\right]$. Finally (3), the tuple $\left[p_{1},\left(q_{1}\right),()\right]$ is deleted.

It is worth noting that the new root $\left[\left(p_{1}\right), q_{1}, r_{1}\right]$ which replaces $\left[p_{1}, q_{1}, r_{1}\right]$ it is not an enablement. In other words, the consolidation graph has lost an enablement as a consequence of the cancellation. This is what we expected to happen, because $p_{1}$ has cancelled its offers because it committed to another interaction. When one enablement of an interaction executes, every conflicting enablement of other or even the same interaction must be refused. In our example, the enablement $\left[p_{1}, q_{1}, r_{1}\right]$ has been refused.

The Process Of fer and the CancelOf fer algorithms are formally proven to be correct in [8]. The correctness proof for the ProcessOffer algorithm relies on proving that the Search algorithm always finds the most consolidated tuples for the input tuple $T$. We can easily prove that if the input tuple $T$ can consolidate with a root of the graph, the result is the most consolidated tuple for that root. And if the consolidation with the root is not possible, it can be recursively proven that the Search algorithm finds the most consolidated tuple under that root. Then, since a tuple having every role consolidated is an enablement, and since every root in the graph is processed, we can prove that the algorithm finds out every enablement originated by an offer. The correctness proof for the Cancel Of fer algorithm relies on proving that it only affects the tuples containing information about the cancelled offer.

\subsection{Optimizing ProcessOffer}

Figure 3 shows the basic enablement detection algorithm. The basic Search algorithm shown in this figure can be optimized in three ways, without loss of correctness.

It is worth noting the costs that those optimizations imply. The first optimization is just an algorithmic criterion,

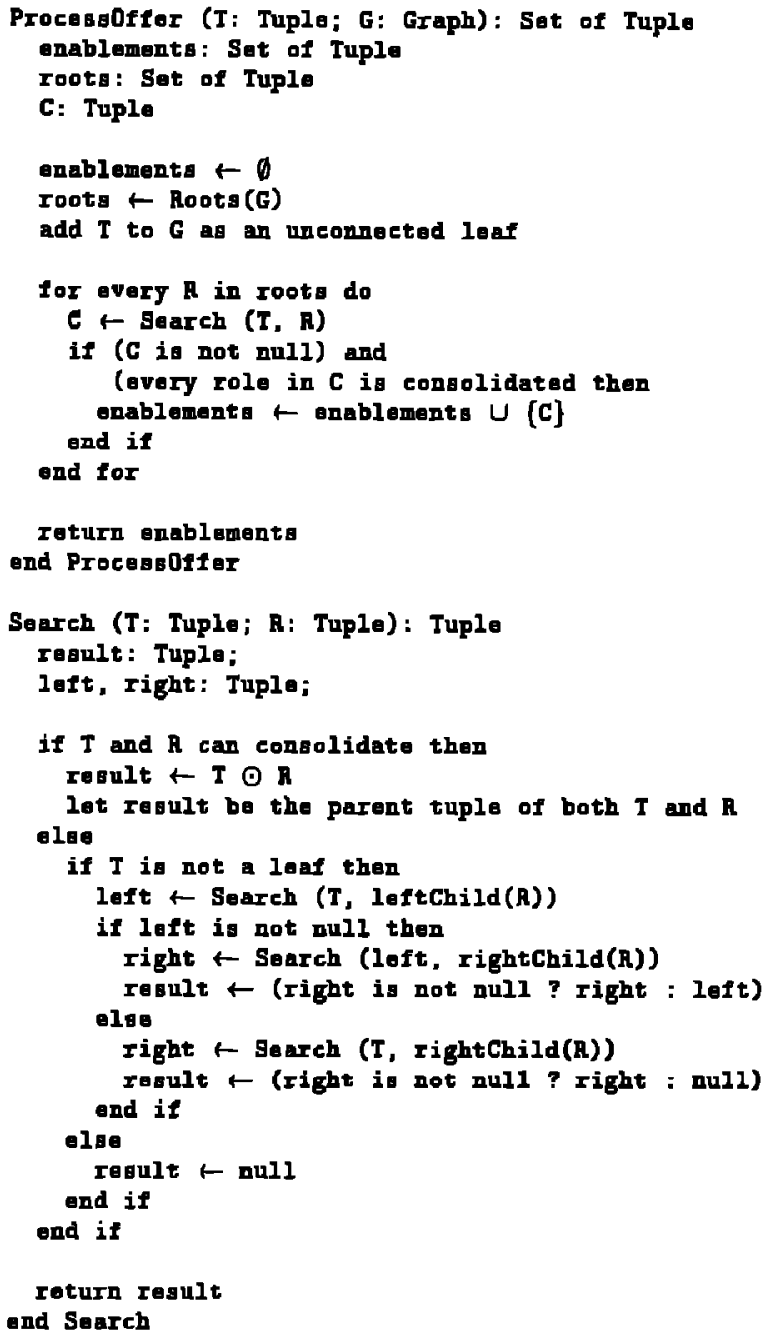

Figure 3: a-solver enablement detection algorithm.

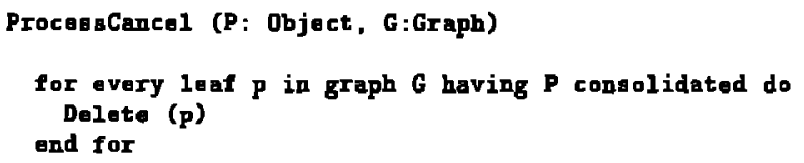

end Delete

Figure 4: $\alpha$-solver cancellation function. 
Rebuild ( $T$ : Tuple)

parents : Set of Tuple

left, right: Tuple

left $\leftarrow$ loftChild $(T)$

right $\leftarrow$ rightChild (T)

Replace ( $T$, left $O$ right)

for every $P$ in Parent $a(T)$ do

Rebuild ( $P$ )

end for

and Rebuild

Figure 5: Recursive rebuild after deletion.

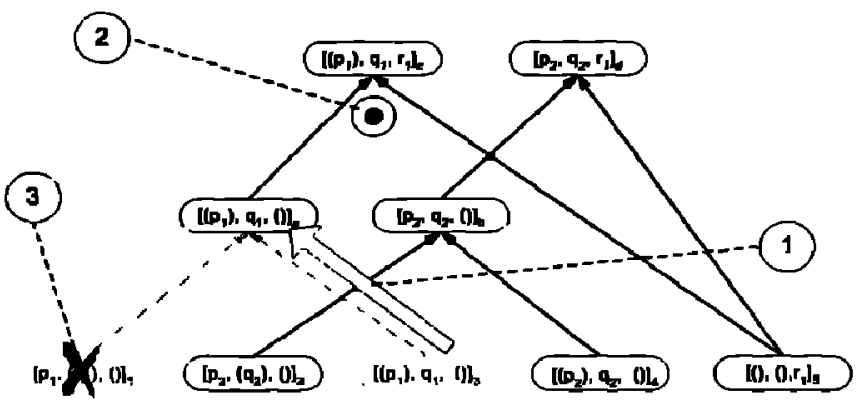

Figure 6: Cancellation of offers from $p_{1}$ in the graph in Figure 2.

having no cost on memory usage. The second optimizations require two boolean and one integer attributes for every tuple, and one offer counter. The third optimization requires the use of indexing functions, that are usually implemented by means of hash tables or sinnilar data structures. Since in some theoretical scenarios this can amount to an important memory consumption, we have implemented it as an optional feature, allowing the operator to decide when it should be performed or when it should not.

\subsubsection{Search Stop}

The first optimisation we can apply relies on the fact that in the Search algorithm, when it tries to consolidate the input tuple $T$ with a $R$ tuple in the graph, and every role in consolidated state in $\boldsymbol{R}$ fails to consolidate with its partner in $T$, there is no tuple $R^{\prime}$ descendant of $R$ that could be consolidated with $T$. Then, no more recursion is needed to process the descendants of $T$.

For example, let us assume that the input tuple $T=$ $\left[p_{1},\left(q_{1}\right),\left(r_{1}\right),\left(s_{1}\right)\right]$ is being checked for consolidation with a tuple $R=\left[(), q_{2}, r_{2}, s_{2}\right]$. Tuple $R$ has consolidated $g_{2}, r_{2}$ and $s_{2}$ in roles $Q, R$ and $S$ respectively. But those three fail to consolidate with $\left(g_{1}\right),\left(r_{1}\right)$ and $\left(s_{1}\right)$. So, it can be proven that there is no tuple among the descendants of $R$ that can be consolidated with $T$.

\subsubsection{Avoiding re-processing of nodes}

The second optimization we propose relies on the fact that the consolidation graph consists of a number of binary trees sharing nodes. Since a node can be reached from more than one root, it may be processed more than once. But, note that in algorithm Search a tuple in the graph can be checked for consolidation (i) with the input tuple $R$ or (ii) with a consolidation of the input tuple $T$ with another tuple in the graph. This happens when a consolidation is found in the graph while processing the left subtree of a tuple, and then a consolidation for it is searched in the right subtree of the tuple. So, we can label every tuple in the graph with a boolean $T$-checked flag that is set to true when the tuple is checked with the $T$ input tuple. The Search algorithm, when is searching a consolidation for the $T$ input tuple, checks the $T$-checked flag of every tuple about to be processed, ignoring the tuples (and its descendants) having this flag set to true. Furthermore, it can be proven that if no consolidation was found under a tuple when processing an offer, regardless of tuple being processed ( $T$ or a consolidation of $T$ with other tuple), no consolidation can be found in a subsequent processing. So, we can label every tuple with a empty boolean flag that is set to true when the tuple is processed and no consolidation is found under it. When a tuple with empty = true is about to be processed, it can be ignored since no consolidation can be found.

Labeling every tuple with attributes has an important drawback, because they must be initialized before every offer is processed. This requires that the whole graph must be traversed, and that is precisely what we need to avoid. A solution to this problem is to label every tuple with an age integer attribute, and using an offer counter that is updated with every offer processed. When a tuple is going to be processed by the Search algorithm, its age attribute is compared with the offer counter. If its age value is smaller than the offer counter, that is the first time that the tuple is being processed for the current offer, so its attributes $T$-checked and empty must initially be set to false. If its age attribute equals the offer counter, that means that the tuple has already been visited along the current offer processing, so its $T$-checked and empty attributes must be taken into account. Note that when the offer counter is about to overflow, it must be reset to zero, and the full graph must be traversed to reset every tuple age attribute. But, if we use 32 or 64 bits for the counters, this is not a problem in practice.

\subsubsection{Starting Search on advantageous nodes}

Finally, the Search algorithm can be optimized making that the search process begin in nodes as close as possible to tuples in the graph that consolidates with the input tuple $T$, instead of beginning the search from the roots of the graph. This optimization relies on the principle that if a $T$ input tuple is like $\left[p_{1}, \ldots\right]$ any tuple that consolidates with it must be like $\left[\left(p_{1}\right), \ldots\right]$ or $[(), \ldots]$. We can prove that when a process of a $p$ participant under role $P$ is being processed, if the search begins (i) in the tuples that require the participant $p$ under role $P$ and (ii) in the tuples that accept any participant under role $P$, such that there is no other tuple above it in the graph that requires or accepts the

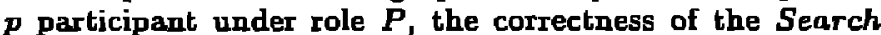
algorithm is preserved.

\section{SELECTION ALGORITHMS}

Once an enablement has been found by the enablement detection algorithm, it must have a chance to be executed. A selection algorithm must ensure exclusion, deciding thus whether each enablement must be selected or refused. An enablement that does not conflict with others should be selected immediately. On the other hand, if an enablement is 
rejected, that is because it conflicts with another one that has already been selected.

Note that since an enablement is determined by a fixed set of participants, the enablement selection problem is analogous to the problem of interaction selection in a static interaction model. since $\alpha$-solver is well-encapsulated into both routines ProcessCancel and Process Of fer, it could work together with any selection algorithm that fulfills the following requirements:

- Coordinators of interactions do not need to be aware one another.

- Participants in an interactions do not need to be aware one another

If coordinators of interactions do not need to be aware one another, there is no problem in that new interactions (enablements) do appear at run-time, since there is no need nor possibility of communication among them. On the other hand, since participants do not necessarily know at compiletime the other participants that are going to participate with them, it is very important that the algorithm do not need the participants to be aware one another.

We have developed an algorithm with fulfills those requirements, an that have been successfully integrated with $\alpha$-solver. This algorithm is called $\alpha$-core, and constitutes together with $\alpha$-solver the implementation of $\alpha$ that we developed for the framework that provides CAL run-time support. A sketch of $\alpha$-core can be found at [7], and the full description and proofs of correctness can be found at [B].

\section{PERFOMANCE}

We have implemented the algorithms ProcessOffer and ProcessCancel described at section 4, and have perform some tests in order to measure their performance. The scenario we have used in our tests is depicted in Figure 7.

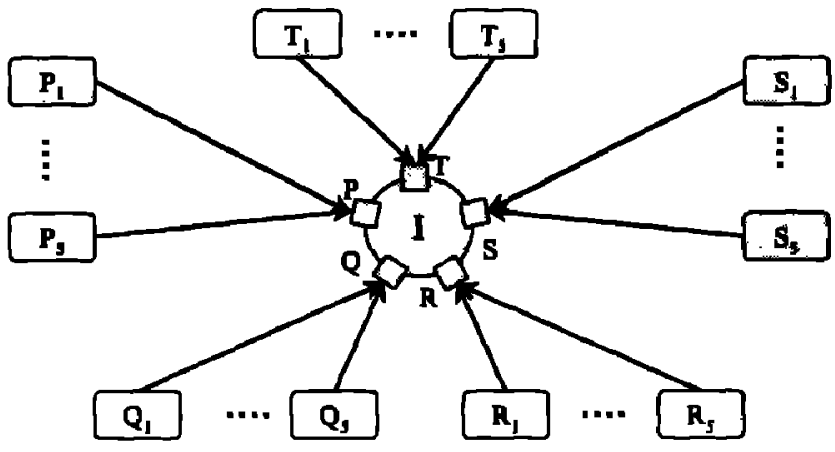

Figure 7: Scenario for testing $\alpha$-solver.

This scenario consists of an interaction $I$ with five roles $P, Q, R, S$ and $T$, and 25 participants, offering five of them to participate under each role in the interaction: participants $P_{1}, \ldots, P_{5}$ offer to participate under rol $P$, participants $Q_{1}, \ldots, Q_{5}$ offer to participate under rol $Q_{1}$ and so on. We have used a five-party interaction because is accepted that greater cardinality interactions have little practical applications [3].

As a measure of our algorithms performance, we have measured the average number of times that the consolida- tion operation $\odot$ is computed amongst two tuples, every time that an offer or a cancellation is processed.

\subsection{Perfomance of ProcessOffer}

To measure how the algorithm Process Offer performs, we have run five tests $T_{1}, \ldots T_{5}$. Test $T_{1}$ is the worgt case for the algorithms, since every participant accepts that every role in the interaction can be played by any other participant. So, $5^{b}=3,125$ enablements are found. In tests $T_{2}, T_{3}, \ldots$ participants make their offers more and more restrictive, imposing a restriction on the participant that must play role $P, Q_{1} \ldots$ and so on, being $T_{5}$ the best case since every participant restricts that every role in the interaction must be played by another concrete participant. So, the number of enablements found in test $T_{2} \ldots T_{5}$ are respectively $5^{4}, 5^{3}, 5^{2}$ and $5^{1}$.

Since the third optimization proposed on Process Offer proposed in section 4.4 algorithm has been implemented as optional, we have run twice every test $T_{1} \ldots T_{5}$ : once with the optimization enabled, and once with this optimization disabled. This permits to compare how good the optimization is. The increase of memory usage due to the optimization has never been greater than $15 \%$.

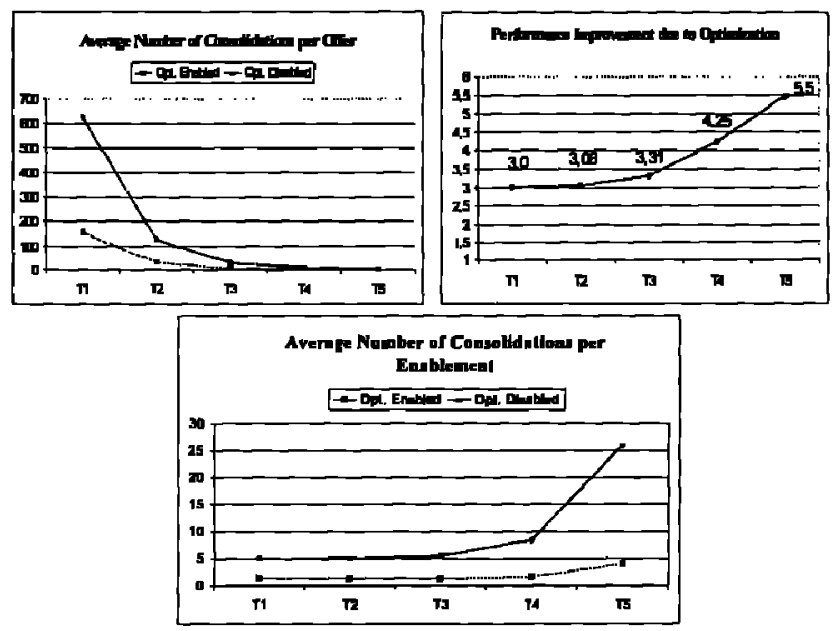

Figure 8: ProcessOffer algorithm performance.

The first plot in Figure 8 shows the average number of consolidation operations computed per offer in each run. We can appreciate that the number of operations computed decreases dramatically when the test is run with the optimization enabled. The second plot shows the relationship amongst the number of operations computed with and without the optional optimization. The improvement due to the optimization increases as the restrictions imposed by the participants are more restrictive, since they provide more information useful to determine where the search process should begin.

Nevertheless, the results of test $T_{1}$ in first plot may seem poor. Is executing an average of 470 or 156 consolidation operations per offer a good performance? Yes, indeed it is, because we should also take into account the average number of enablements found per offer. The third plot in Figure 8 shows the average number of consolidation operations computed per enablement found, as much in the test with and 
without the optional optimization. This gives us the cost of finding an enablement. So, in test $T_{1}$ the average cost of finding an enablement is less than four consolidation operations if the optional optinization is not enabled, and less than two otherwise. It is very important noting that the cost of finding an enablement decreases as the number of enablements that can be found increases.

\subsection{Perfomance of CancelOf fer}

To measure how the algorithm Cancel $O$ f $f$ er performs, we have run again the same five tests $T_{1}, \ldots T_{5}$ from previous section, cancelling every offer after each run.
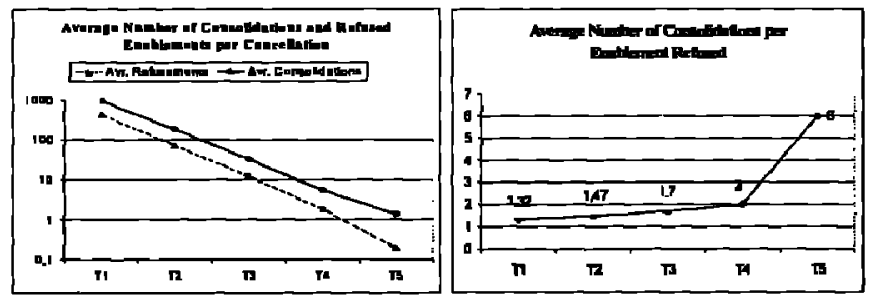

Figure 9: CancelOffer algorithm performance.

The first plot in Figure 9 shows the average number of consolidation operations computed per cancellation, and the average number of enablements refused each time. As in the Process Offer algorithm, the numbers in test $T_{1}$ may seem excessively high. But once again, we should take into account the number of enablements refused by every cancellation. The second plot in the Figure shows the average number of consolidation operations cornputed per enablement refused. This is the cost of refusing an enablement. As for ProcessOffer algorithm, we can see how the cost of refusing an enablement decreases as the number of enablements increases. We can find the explanation for this effect in the consolidation graph topology. When there are many enablements in the graph, frequently many of those enablements share leaves nodes. When an offer having many enablements as ancestors in the graph is canceled, the cost of refusing those enablements is smaller that if the tuple had one or none enablements as ancestors.

\section{CONCLUSIONS}

In this paper, we have described the algorithms we have developed to implement the CAL interaction model, focusing on the problem of enablement detection. This is an open multiparty model useful for applications that require coordination amongst entities that are not fixed beforehand. Although the problem of finding all the sets of entities that agree to coordinate through an interaction has a high computational cost, our algorithms performs quite well since it behaves more efficiently as the complexity increases.

Our enablement detection algorithm can cooperate with any selection algorithm that fulfills some conditions. We think that this is an important feature, because it leaves an open door to deal with other problems related to selection, such as fairness.

\section{APPENDIX}

\section{A. REFERENCES}

[1] R. Bagrodia. Process synchronization: Design and performance evaluation of distributed algorithms. IEEE Transactions on Software Engineering, 15(9):1053-1065, Sept. 1989.

[2] R. Corchuelo, J. Pérez, and M. Toro. A Multiparty Coordination Aspect Language. ACM SIGPLAN, 35(12):24-32, Dec. 2000.

[3] N. Francez and I. Forman. Interactirig processes: $A$ multiparty approach to coordinated distributed programming. Addison-Wesley, 1996.

[4] Y. Joung. A comprehensive study of the complexity of multiparty interaction. In Proceedings of the $19^{\text {th }}$ Annual ACM Symposium on Principles of Programming Languages POPL'g2, pages 142-153. ACM Press, Jan. 1992.

[5] Y. Joung and S. Smolka. A completely distributed and message-efficient implementation of synchronous multiprocess communication. In P.-C. Yew, editor, Proceedings of the $19^{\text {th }}$ International Conference on Parallel Processing. Volume 3: Algorithms and Architectures, pages 311-318, Urbana-Champaign, Illinois, Aug. 1990. Pennsylvania State University Press.

[6] Y. J. Joung and S. A. Smolka. Strong interaction fairness via randomization. In Proceedings of the $16^{\text {th }}$ International Conference on Distributed Computing Systems, pages 475-483, Hong Kong, May 1996. IEEE Computer Society Press.

[7] J. Pérez, R. Corchuelo, D. Ruiz, and M. Toro. A framework for aspect-oriented multiparty coordination. In New Developments in Distributed Applications and Interoperable Systems, pages 161-174. Kluwer Acadernic Publishers, 2001.

[8] J. A. Pérez. Un Framework Orientado a Aspectos para la Descripción del Comportamiento Coordinado. Aplicación a los Sistemas Multiorganizacionales. PhD thesis, Facultad de Informática y Estadística. Dpto. de Lenguajes y Sibtemas Informáticos. Universidad de Sevilla, 2001.

[9] Y. Tsay and R. Bagrodia. A real-time algorithm for fair interprocess synchronization. In Proceedings of the $12^{\text {th }}$ International Conference on Distributed Computing Systems, pages 716-723, Washington, D.C., USA, June 1992. IEEE Computer Society Press. 